\title{
Microwave tissue coagulation technique in anatomical liver resection
}

\author{
KAI TAN, XILIN DU, JIKAI YIN, RUI DONG, LI ZANG, TAO YANG and YAFENG CHEN \\ Department of General Surgery, Second Division, TangDu Hospital Affiliated to the \\ Fourth Military Medical University, Xi'an, Shaanxi 710038, P.R. China
}

Received October 30, 2013; Accepted December 16, 2013

DOI: $10.3892 /$ br.2014.227

\begin{abstract}
Anatomical liver resection is currently the preferred treatment for liver cancer. With the recent introduction of medical microwave coagulation for liver metastases, anatomical hepatectomy may be performed more efficiently. The present study retrospectively reviewed the results of microwave tissue coagulation performed during anatomical liver resection for patients with liver disease at the TangDu Hospital (Xi'an, China) between January, 2009 and June, 2012. A total of 128 patients met the inclusion criteria and were divided into two groups for comparison; those treated with the microwave coagulation technique $(n=66)$ and the conventional group $(n=62)$, who were treated with standard partial hepatectomy. There was no reported perioperative mortality. The univariate analysis revealed that the duration of liver dissection, intraoperative blood loss, intraoperative erythrocyte transfusion volume and alanine aminotransferase levels on the 5 th postoperative day were significantly different between the microwave and conventional groups $(\mathrm{P}<0.05)$. Therefore, microwave tissue coagulation in anatomical liver resection was shown to be efficacious and safe and, provided proficient skills are developed in this technique, microwave tissue coagulation may be an effective alternative to anatomical hepatectomy.
\end{abstract}

\section{Introduction}

Anatomical liver resection is currently the preferred treatment for liver cancer. The procedure involves total resection of the nidus, preserves a large amount of normal liver tissue and minimizes blood loss $(1,2)$. A diverse number of instruments and devices are currently available for use in liver resection, each exhibiting advantages and disadvantages: Cut-Ultrasound Aspiration (CUSA), High-Frequency Electrosurgical Knife, Ultrasound Cutter, Microwave Tissue

Correspondence to: Dr Xilin Du, Department of General Surgery, Second Division, TangDu Hospital Affiliated to the Fourth Military Medical University, 1 New Temple Road, Xi'an, Shaanxi 710038, P.R. China

E-mail: dxlin0705@163.com

Key words: microwave tissue coagulator, anatomical liver resection, liver cancer, surgical technique
Coagulator, Helix Hydro-jet, Tissue Link, Argon Scalpel and Radio Frequency Cutter, are the most commonly used. With the recent development of medical microwave coagulation, along with improved techniques and skill acquisition, hepatectomy may be performed more efficiently in patients with liver disease $(3,4)$. In the present study, we collected and retrospectively analyzed the clinical data from 128 patients with liver disease who were surgically treated with anatomical hepatectomy and either microwave coagulation therapy $(n=66)$ or standard partial hepatectomy $(n=62)$ between January, 2009 and June, 2012 at the TangDu Hospital Affiliated to the Fourth Military Medical University (Xi'an, China). The aim of the study was to compare the efficacy and safety of microwave coagulation to that of conventional liver resection in patients with liver disease.

\section{Materials and methods}

Patients, groups and etiology. This study was approved by the Ethics Committee of the TangDu Hospital. Informed consent was obtained from all the recruited subjects. A total of 332 patients were treated with hepatectomy in our hospital between January, 2009 and June, 2012. Following the exclusion of patients with irregular hepatectomy $(n=150), 32$ patients were treated with microwave ablation, radiofrequency ablation and/or transcatheter arterial chemoembolization prior to liver resection. A second hepatectomy was performed in 13 patients. Five patients without liver cirrhosis and four patients developed acute liver failure postoperatively. A total of 128 patients met the inclusion criteria and were divided into two groups based on whether the microwave tissue coagulator technique was used intraoperatively (Table I). The microwave tissue coagulator group (microwave group) included 66 patients and the conventional group included 62 patients treated by anatomical liver resection with conventional surgical instruments. The recruited patients included 107 men and 21 women, with an average age of 50.3 \pm 10.0 years (range, $25-77$ years). The cause of liver disease was hepatitis B-related cirrhosis in 119 patients (93\%), hepatic cirrhosis with hepatitis C and B simultaneously or hepatitis $\mathrm{C}$ in seven patients $(5.5 \%)$ and alcoholic cirrhosis in two patients $(1.5 \%)$. All the patients underwent selective surgery in a preoperative optimal medical condition. A total of $8 \%$ of the cases classified as Child-Turcotte-Pugh (CTP) B, 
Table I. Couinaud classification ${ }^{\mathrm{a}}$ of resected liver segments in patients treated with microwave coagulation or conventional methods.

\begin{tabular}{lcc}
\hline Classification & $\begin{array}{c}\text { Microwave group } \\
(\mathrm{n}=66)\end{array}$ & $\begin{array}{c}\text { Conventional group } \\
(\mathrm{n}=62)\end{array}$ \\
\hline I & 0 & 2 \\
II & 1 & 0 \\
III & 3 & 2 \\
IV & 2 & 3 \\
V & 5 & 2 \\
VI & 4 & 7 \\
VII & 6 & 4 \\
VIII & 3 & 6 \\
II+III & 7 & 4 \\
III+IVb & 0 & 1 \\
IVb+V & 4 & 1 \\
V+VI & 7 & 9 \\
V+VIII & 1 & 2 \\
VI+VII & 8 & 5 \\
VII+VIII & 5 & 3 \\
I+IV+V & 0 & 1 \\
II+III+IV & 3 & 4 \\
IVb+V+VI & 1 & 0 \\
V+VI+VII & 2 & 0 \\
V+VI+VII+VIII & 4 & \\
& & \\
& &
\end{tabular}

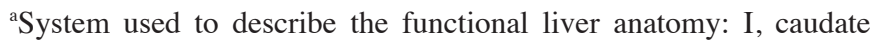
lobe; II, superior subsegment of the lateral segment; III, inferior subsegment of the lateral segment; IV, medial segment; V, inferior subsegment of the anterior segment; VI, inferior subsegment of the posterior segment; VII, superior subsegment of the posterior segment; VIII, superior subsegment of the anterior segment; IVb, inferior subsegment of the medial segment.

ameliorated to CTP A following pharmacotherapy. The indocyanine green retention rate at 15 min was $<12 \%$ in $89.2 \%$ of the patients.

Microwave tissue coagulator and surgical technique. The surgical procedures were performed by the same operating team. Microwave tissue coagulation consisted of two parts (Fig. 1): the first used the FORSEA MTC-3C microwave ablation system (Qinghai Microwave Electronic Institute, Nanjing, China), with an output power of $2,450 \pm 50 \mathrm{MHz}$ continuous wave and a range of 0-100 W (80-100 W was used intraoperatively, with construction of the coagulation belt at 80 and $100 \mathrm{~W}$ for hematischesis) that can coagulate a vascular area of $5 \mathrm{~mm}$ in diameter. The second part utilized the Cool-Circle microwave applicator or antenna (actual needle length of $180 \mathrm{~mm}$ with an external diameter of $14 \mathrm{G}$, with a needle tip releasing power), which can ablate a coagulation zone to an optimal yellow-white color after 10-20 sec, up to a diameter of $10 \mathrm{~mm}$, with a stitch length of $15-20 \mathrm{~mm}$. No radiation was applied outside a range of 3-5 $\mathrm{cm}$. Hepatic segmental resection according to the Couinaud criteria (5) was performed following identification of the large segmental vessels with B-ultrasound delineation. For large tumors compressing liver tissue, the identification of the segments was not clear and the anatomical boundaries were marked by injection of methylthioninium chloride under B-ultrasound guidance. There was no control of the porta hepatis in the microwave group during the procedure; however, 53 cases were controlled from the conventional group (left or right according to the Glisson system). The liver segment occupied by the tumor was removed through the midline of the coagulated area by an electro-scalpel or after the initial coagulative puncta, the tissue was dissected whilst cauterizing the next puncta (Fig. 2).

Statistical analysis. Multiple factors were used in the comparative analysis, including age, gender, preoperative alanine aminotransferase (ALT) levels, hepatectomy classification (classified into three groups: resection of 1,2, or $\geq 3$ segments), duration of liver dissection, intraoperative blood loss, intraoperative erythrocyte transfusion volume, ALT levels on the 1st, 3rd and 5th postoperative days, postoperative drainage volume from the abdominal cavity on the 2 nd, 5 th and 7 th days, postoperative liver function recovery time (return to CTP A), postoperative cumulative time of fever and postoperative complications (biliary fistula and hydrothorax). The measurement data are expressed as the means \pm standard deviation following statistical analysis with the t-test. Enumeration data were analyzed with the $\chi^{2}$ test for $\mathrm{RxC}$ tables or the Fisher's exact test if beyond the $\chi^{2}$ test. SPSS software, version 13.0 for Windows (SPSS, Inc., Chicago, IL, USA) was used for analyses and the results were considered significant for levels of $\alpha=0.05$.

\section{Results}

Postoperative outcome and histological examination. There was no reported perioperative mortality. The average hepatic portal occlusion time was $13.7 \pm 5.2 \mathrm{~min}$ in the conventional group with closure of the section of remnant liver in 41 cases; however, no sections were closed in the microwave group. Following histological examination, 115 of the 128 cases were diagnosed with hepatocellular carcinoma, six with combined hepatocellular and intrahepatic cholangiocarcinoma, three with intrahepatic cholangiocellular carcinoma, two with clear cell hepatocellular carcinoma and two with colon cancer liver metastasis.

Postoperative complications. Five patients developed a postoperative biliary fistula and three had a large hemorrhage (controlled with medication in two patients and further surgery in one case with bleeding from the right inferior phrenic artery, no one bleeding from sections of the liver remnants). There were no patients with evidence of hyperpyrexia and the mean cumulative time of reported fever in the microwave group was $0.9 \pm 0.8$ days, with the majority of patients developing a low-grade fever on the 2 nd and 3rd postoperative day $(52.1 \%$ of all cases with fever) in the microwave group. The mean cumulative time of reported fever was $1.1 \pm 0.9$ days in the conventional group $(\mathrm{P}=0.196)$ (Table II).

Comparison between the microwave coagulation and conventional liver resection groups. The univariate analysis revealed 

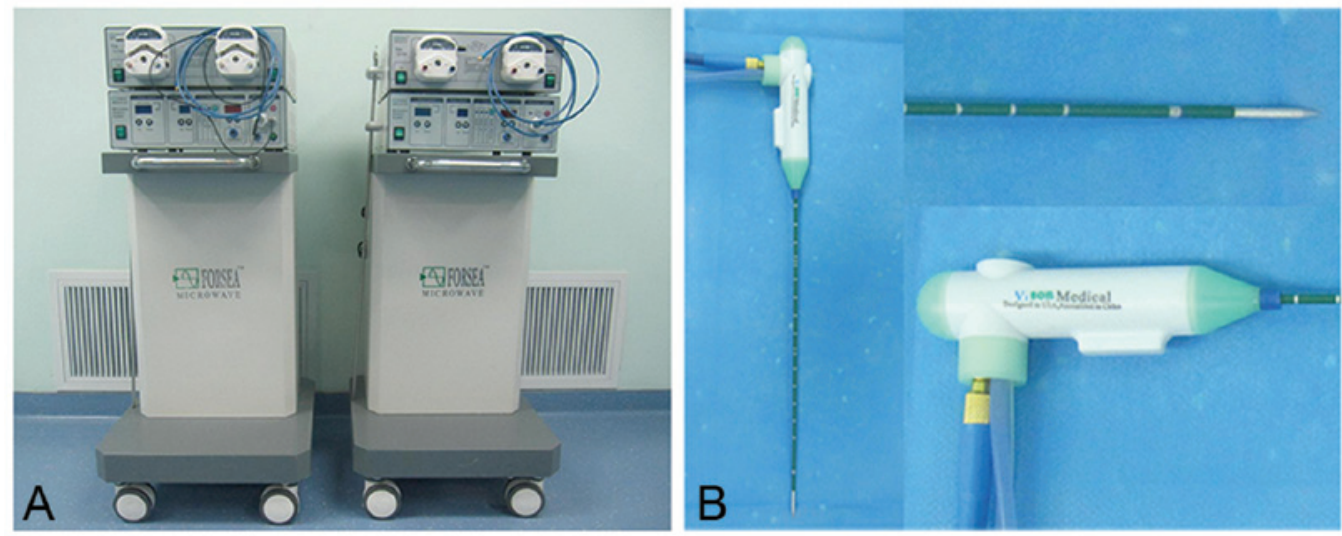

Figure 1. (A) Microwave ablation system. (B) Cool-Circle microwave applicator.

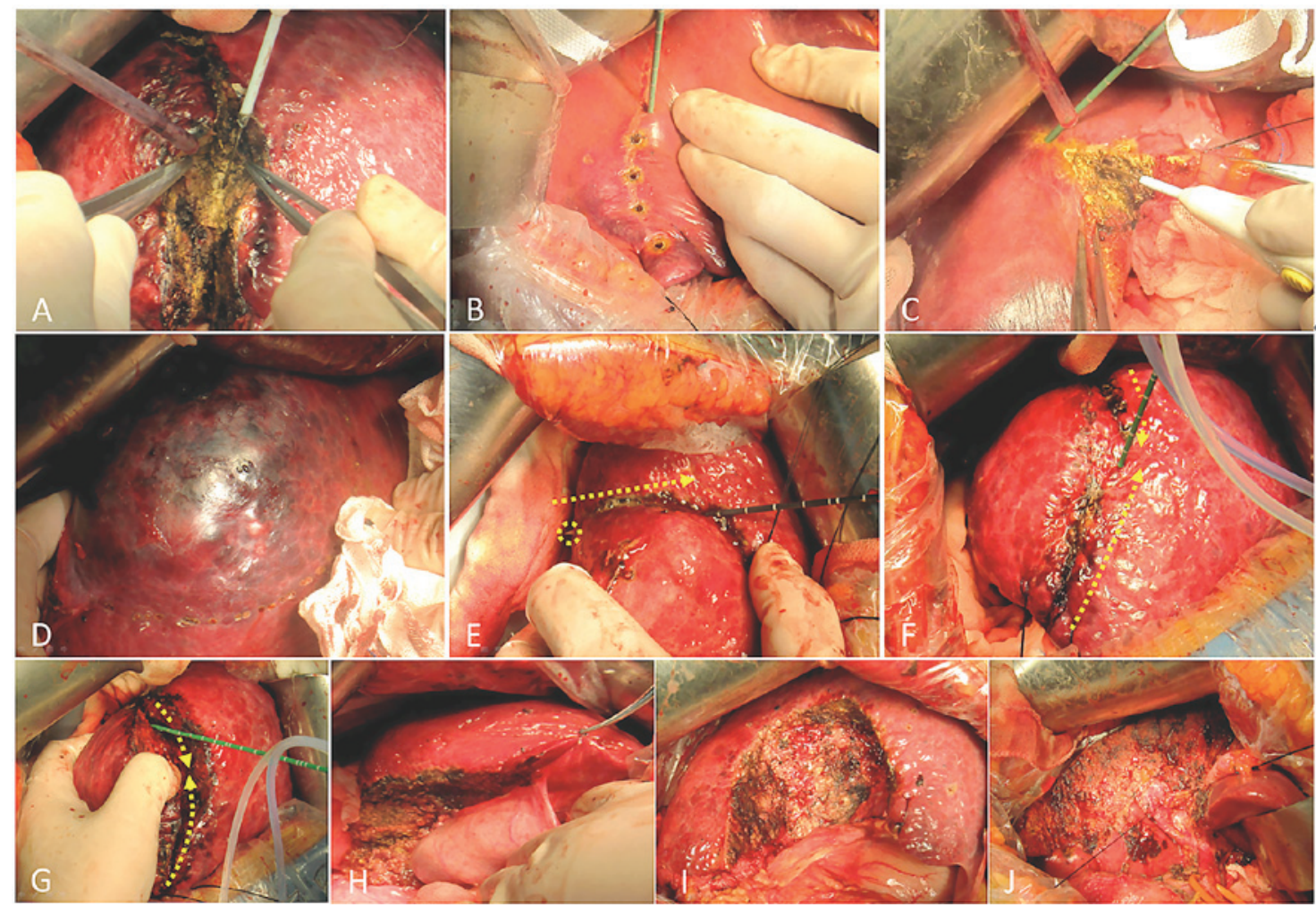

Figure 2. Intraoperative procedure of anatomical hepatectomy. (A) Dissection was performed through the midline of a yellow-white coagulation belt. (B) To ablate the coagulation belt, coagulative puncta of $10 \mathrm{~mm}$ in diameter were performed in 10-20 sec and the stitch length was $\sim 15-20 \mathrm{~mm}$. (C) After the first coagulative puncta, the tissue was dissected whilst cauterizing the next puncta. (D) Segment VII was marked by injection of methylthioninium chloride. (E) The antenna tip was isolated with gauzes to prevent side impairments. At the fringe of the liver, the tissue was penetrated by the microwave antenna, followed by tissue coagulation, antenna withdrawal and continuous coagulation, until a successive coagulated zone was completed. (F) A successive zone was ablated from the fringe to the middle along the pre-excision line. (G) Following discission of the superficial zone, deep coagulation from the fringe to the middle was performed. (H) Remnant liver following removal of segments VI+VII. (I) Segment V removed. (J) Segments IVb+V+VI removed.

that age, gender, preoperative ALT levels, hepatectomy classification, ALT levels on the 1st and 3rd postoperative day, postoperative drainage volume of the abdominal cavity, postoperative liver function recovery time (recovery to CTP B or A), postoperative cumulative time of fever, biliary fistula and hydrothorax were not significantly different between the two groups $(\mathrm{P}>0.05)$. However, there were significant differences in the duration of liver dissection, intraoperative blood loss, intraoperative erythrocyte transfusion volume and ALT levels on the 5 th postoperative day $(\mathrm{P}<0.05)$ between the two groups: the microwave group required a longer operative time, but was associated with less intraoperative blood loss and reduced blood transfusion requirements compared to the conventional group (Table II).

\section{Discussion}

The current procedure for liver surgery is based on the principle described by Couinaud (5), stating that the liver is divided into eight functional segments. Accordingly, each 
Table II. Outcomes of anatomical hepatectomy in the microwave coagulation and the conventional groups.

\begin{tabular}{|c|c|c|c|c|}
\hline Factors & $\begin{array}{l}\text { Microwave } \\
\text { group }(n=66)\end{array}$ & $\begin{array}{l}\text { Conventional } \\
\text { group }(n=62)\end{array}$ & $t / \chi^{2}$ & P-value \\
\hline Age, years & $50.7 \pm 9.9$ & $49.9 \pm 10.2$ & 0.403 & 0.688 \\
\hline Gender, male & $52(78.8 \%)$ & $55(88.7 \%)$ & 2.295 & 0.130 \\
\hline Preoperative ALT levels, U/1 & $20 \pm 7.2$ & $18 \pm 6.8$ & 1.613 & 0.109 \\
\hline \multicolumn{5}{|l|}{ Intraoperative status of liver dissection } \\
\hline Hepatectomy classification & - & - & 0.863 & 0.649 \\
\hline Resection of 1 segement & $24(36.4 \%)$ & $26(41.9 \%)$ & - & - \\
\hline Resection of 2 segements & $32(48.5 \%)$ & $25(40.3 \%)$ & - & - \\
\hline Duration, min & $61 \pm 31$ & $48 \pm 25$ & 2.603 & 0.010 \\
\hline Hemorrhage, ml & $245 \pm 102$ & $355 \pm 171$ & -2.052 & 0.042 \\
\hline Erythrocyte transfusion, min & $150 \pm 67$ & $215 \pm 112$ & -4.012 & $<0.001$ \\
\hline \multicolumn{5}{|l|}{ Postoperative ALT levels, U/l } \\
\hline Day 1 & $458 \pm 387$ & $408 \pm 302$ & 0.811 & 0.419 \\
\hline Day 3 & $296 \pm 212$ & $358 \pm 274$ & -1.435 & 0.154 \\
\hline Day 5 & $108 \pm 71$ & $236 \pm 204$ & -4.794 & $<0.001$ \\
\hline \multicolumn{5}{|c|}{ Drainage volume of the abdominal cavity, $\mathrm{ml}$} \\
\hline Day 2 & $110 \pm 88$ & $135 \pm 117$ & -1.371 & 0.173 \\
\hline Day 5 & $25 \pm 19$ & $30 \pm 24$ & -1.312 & 0.192 \\
\hline Day 7 & $16 \pm 13$ & $20 \pm 15$ & -1.615 & 0.109 \\
\hline Liver function recovery time (days) & $4.3 \pm 3.8$ & $5.1 \pm 4.2$ & -1.132 & 0.260 \\
\hline Cumulative time of fever (days) & $0.9 \pm 0.8$ & $1.1 \pm 0.9$ & -1.299 & 0.196 \\
\hline \multicolumn{5}{|l|}{ Complications } \\
\hline Biliary fistula & $2(3.0 \%)$ & $3(4.8 \%)$ & a & 0.673 \\
\hline Hydrothorax & $16(24.2 \%)$ & $18(29.0 \%)$ & 0.376 & 0.540 \\
\hline
\end{tabular}

Values are presented as means \pm standard deviation or as numerical value (percentage). ${ }^{a}$ Fisher's exact test. ALT, alanine aminotransferase.

segment possesses its own portal vein, hepatic artery and biliary tract, with potential interspace between two adjacent segments. Anatomical hepatectomy involves resection of a segment, sector, hemiliver or trisector (II+III+IV+V+VIII \pm I, or $\mathrm{IV}+\mathrm{V}+\mathrm{VI}+\mathrm{VII}+\mathrm{VIII} \pm \mathrm{I})(5)$. For patients with cirrhosis and liver cancer, anatomical hepatectomy combines radical excision of the nidus with low levels of blood loss and the highest possible preservation of liver tissue. Recent technological advances have allowed surgeons to use novel instruments and devices for liver resection, including CUSA, High-Frequency Electrosurgical Knife, Ultrasound Cutter and Microwave Tissue Coagulator.

The application of microwave fields in hepatic surgery was first used in the 1970s. Successive punctures with a monopolar antenna create a continuous zone allowing hepatic dissection in the midline with reduced blood loss. The resonance of hydrogen and polar material from a microwave field heat tissue via mutual friction between hydrogen and hydrogen, polar material and its counterpart and hydrogen and polar material; subsequently, the antenna produces protein coagulation (6).

Intraoperative hemorrhage is one of the most significant factors associated with prognosis and the complications of liver surgery $(7,8)$; therefore, a number of surgeons prefer the microwave coagulator for controlling hematischesis $(9,10)$.
The present study demonstrated that the duration of liver dissection, intraoperative blood loss and intraoperative erythrocyte transfusion volume were significantly different between the groups treated with microwave coagulation and conventional techniques $(\mathrm{P}<0.05)$. As the microwave coagulator is theoretically capable of coagulating vessels of $5 \mathrm{~mm}$ in diameter, hepatectomy with mini-hemorrhagic spots following dissection of the coagulation area may result in less transfixion or coagulation of duct stumps during liver transection, thus simplifying the procedure. Therefore, surgeons are able to concentrate on the manipulation of the large vessels, reducing the overall blood loss. Generally, with more mini-hemorrhagic spots occurring in the conventionally treated group, while the porta hepatis is controlled, the surgeons may perform liver dissection and manipulate hemorrhagic spots at a faster rate, resulting in a relatively poor hematischesis control.

Following opening of the porta hepatis, the increased transfixion or occlusion of duct stumps requires a certain degree of hematischesis. The key reasons for blood loss in patients treated with the microwave coagulator are occlusion of hepatic vein branches and disconnection of the lateral branches around the liver in patients with cirrhosis.

With a larger coagulation area, large vessel branches of vascular deep tissue become exposed following dissection of 
the yellow-white belt, causing cirrhotic liver tissue to be more firm during dissection, with fragile vascular structures; therefore, gentle manipulation and dissection is required, with extra caution during the manipulation of the duct stumps, possibly leading to surgeon fatigue in the conventional group.

This study demonstrated that the duration of liver dissection was significantly less in the conventional group compared to that in the microwave group. In the conventional group, surgeons performed liver resection more rapidly to shorten the duration of interruption of the porta hepatis flow during control. However, this was offset due to the longer time spent on coagulation.

Following the completion of the liver dissection in the conventional group, the surgeons spent more time treating hemorrhagic spots in liver sections. B-ultrasound was used to locate large vessels and the anatomical boundaries of resection, as well as to check the entire liver and eliminate any micro-nidus and/or suspected nidus. Using the microwave coagulator, this procedure may be performed with more finesse. Previous studies reported that the application of microwave coagulation in unresectable liver cancer may be performed safely, with a significant overall benefit $(11,12)$.

During surgery, we observed the following: i) liver tissue is more fragile compared to the large ducts near the porta hepatis, while resistance may be felt when the microwave antenna punctures the duct wall; ii) the antenna tip should be isolated with gauze or other tools to prevent side effects; iii) at the fringe of the liver, which is free of large vascular branches, the tissue should be penetrated a little, then coagulated, followed by antenna withdrawal and continuous coagulation; the superficial zone should be ablated from the fringe to the middle, until a successive coagulative zone is completed, whereas the deep belt is created by the same procedure; iv) for the hematischesis of the liver sections, the antenna should be inserted into the section near the hemorrhagic spots, but not directly penetrate the spots; and v) although microwave coagulation is theoretically capable of blocking a 5-mm duct, for ducts $<3 \mathrm{~mm}$ in diameter, coagulation was selected, whereas for ducts $>3 \mathrm{~mm}$ in diameter, ligation and transfixion are considered to be the optimal methods.

The quality of hepatectomy is advanced based on proficient skills on new instruments and tools; however, this may be achieved only after mastering conventional techniques and thoroughly comprehending liver anatomy.

In our study, the ALT levels on the 5th postoperative day were significantly different between the two groups, possibly due to the impairment of transfixed tissue following closure of remnant liver segments in the conventionally treated group. Microwave coagulation may simplify liver surgery techniques by avoiding hepatic portal occlusion and section closing.

Following anatomical hepatectomy, the CTP score may be affected by several factors, including large hemorrhage, poor compensation of liver function and a reduction in the amount of normal tissue. However, the liver function recovery time was not significantly different between the two groups, which is possibly due to the procedure affecting liver function, postoperative pharmacological management and nutritional support.

The use of microwave technology in liver surgery has been associated with only a few complications $(13,14)$. In our study, postoperative fever was not significantly different between the two groups and it was only a low-grade fever, easily manageable with medical treatment. Compared to other tools used in liver surgery, the histopathological examination of the resected specimens from the microwave group revealed more prominent edge necrosis (6). Therefore, caution is advised when inserting the microwave needle, particularly when closing large vascular structures. The bile duct is the largest structure of the Glisson system. Our study revealed no significant differences between the two groups in terms of biliary fistula formation or large hemorrhages. The histopathological examination of liver cross-sections revealed a superior efficacy of the microwave coagulation technique for duct stump closure.

In conclusion, we demonstrated that microwave tissue coagulation for anatomical hepatectomy is efficacious and safe for the ablation of any micro-nidus and/or potentially malignant nidus under B-ultrasound guidance. With appropriate training and skills, the quality of hepatectomy may be improved with microwave coagulation. However, there are certain disadvantages to microwave coagulation: the limited use of this technique outside of research centers and the requirement for technological improvements, with more rapid coagulation and less destruction of adjacent tissues. The limitations of this study lie with its retrospective nature. Further investigation is required in a prospective study, with careful matching of the two groups for the type of resection performed.

\section{Acknowledgements}

The authors would like to thank the device team, including Li Zang, Yin Duan, Zhi-peng Yu and Lu Wang for the photograph collection and maintenance of the equipment. This study was supported by a grant from the National Natural Science Foundation of China (no. 81172287).

\section{References}

1. Yin DL, Jiang HC, Liang YJ, Meng XZ, Wang JB, Zheng TS and Liu LX: Precise hepatectomy guided by minimally invasive surgery: a novel strategy for liver resection. Hepatogastroenterology 59: 1951-1959, 2012.

2. Guglielmi A, Ruzzenente A, Conci S, Valdegamberi A and Iacono C: How much remnant is enough in liver resection? Dig Surg 29: 6-17, 2012.

3. Percivale A, Griseri G, Gastaldo A, Benasso M and Pellicci R: Microwave assisted liver resection: clinical feasibility study and preliminary results. Minerva Chir 67: 415-420, 2012.

4. Inokuchi R, Seki T, Ikeda K, et al: Percutaneous microwave coagulation therapy for hepatocellular carcinoma: Increased coagulation diameter using a new electrode and microwave generator. Oncol Rep 24: 621-627, 2010.

5. Couinaud C: Liver anatomy: portal (and suprahepatic) or biliary segmentation. Dig Surg 16: 459-467, 1999.

6. Tabuse K: Basic knowledge of a microwave tissue coagulator and its clinical applications. J Hepatobiliary Pancreat Surg 5: 165-172, 1998.

7. Kappa SF, Gorden DL, Davidson MA, Wright JK and Guillamondegui OD: Intraoperative blood loss predicts hemorrhage-related reoperation after orthotopic liver transplantation. Am Surg 76: 969-973, 2010.

8. Yildirim IO, Salihoglu Z, Bolayirli MI, Colakoglu N and Yuceyar L: Prospective evaluation of the factors effective on morbidity and mortality of the patients having liver resection surgeries. Hepatogastroenterology 59: 1928-1932, 2012.

9. Imura S, Shimada M, Utsunomiya T, et al: Ultrasound-guided microwave coagulation assists anatomical hepatic resection. Surg Today 42: 35-40, 2012. 
10. Nanri M, Udo K, Kawasaki M, et al: Microwave tissue coagulator induces renal apoptotic damage to preserved normal renal tissue following partial nephrectomy. Clin Exp Nephrol 13: 424-429, 2009.

11. Itoh S, Ikeda $\mathrm{Y}$, Kawanaka $\mathrm{H}$, et al: Efficacy of surgical microwave therapy in patients with unresectable hepatocellular carcinoma. Ann Surg Oncol 18: 3650-3656, 2011.

12. Martin RC, Scoggins CR and McMasters KM: Safety and efficacy of microwave ablation of hepatic tumors: a prospective review of a 5-year experience. Ann Surg Oncol 17: 171-178, 2010.
13. Livraghi T, Meloni F, Solbiati L and Zanus G; Collaborative Italian Group using AMICA system: Complications of microwave ablation for liver tumors: results of a multicenter study. Cardiovasc Intervent Radiol 35: 868-874, 2012.

14. Veltri A, Gazzera C, Rotondella C, Camerano F, Busso M and Gandini G: Image-guided microwave ablation of hepatic tumours: preliminary experience. Radiol Med 117: 378-392, 2012. 